\title{
In vitro culture of primary human myoblasts by using the dextran microcarriers Cytodex $3^{\circledast}$
}

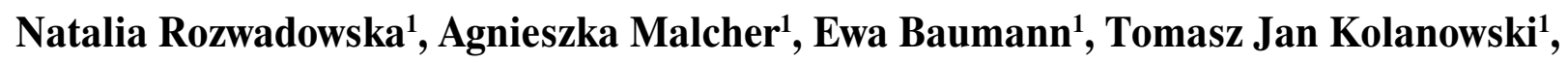 \\ Marek Rucinski ${ }^{2}$, Tomasz Mietkiewski², Katarzyna Fiedorowicz ${ }^{1}$, Maciej Kurpisz ${ }^{1}$
}

${ }^{1}$ Department of Reproductive Biology and Stem Cells, Institute of Human Genetics Polish Academy of Sciences, Poznan, Poland

${ }^{2}$ Traumatology and Orthopaedics Department, District Hospital of Wielkopolska, Poznan, Poland

\begin{abstract}
Introduction. Primary cells in vitro culture scale-up is a crucial issue in cell-based tissue and organ regeneration therapy. Reducing costs and space occupied by the cells cultured in vitro has been an important target. Cells cultured in vitro with the use of bioreactor with dextran microcarriers $\left(\right.$ Cytodex $\left.^{\circledR}\right)$ have potentially a chance to meet many of the cell therapy requirements.

Material and methods. We used collagen-coated carriers (Cytodex $3^{\circledast}$ ) and a spinner flask bioreactor to develop environment suitable for human myoblast proliferation. In parallel, standard adherent in vitro culture conditions for myoblasts propagation (T-flask) were conducted. Cell cycle characterization, senescence, myogenic gene expression and cell apoptosis were evaluated in order to find differences between two culture systems under study. Results. The number of cells obtained in bioreactor per $10^{6}$ of starting cells population was approximately ten times lower in comparison with T-flask culture system. The microcarriers cultured adult myoblasts in comparison with the regular T-flask culture showed faster and more advanced replicative aging and lower proliferative potential. Moreover, the percentage of the cells that entailed an irreversible cell arrest (G0 phase) was also significantly $(\mathrm{p}<0.0001)$ increased.

Conclusions. Our results suggest that population of primary human myoblasts obtained from adult individuals and propagated on dextran microcarriers did not meet the requirements of the regenerative medicine regarding quantity and quality of the cells obtained. Nonetheless, further optimization of the cell scaling up process including both microcarriers and/or bioreactor program is still an important option. (Folia Histochem Cytobiol. 2016, Vol. 54, No. 2, 81-90).
\end{abstract}

Key words: human myoblasts; bioreactor; T-flask culture; stem cell precursors; cell senescence; telomeres; myogenic gene expression; cell cycle

\section{Introduction}

Cell populations prepared for the purpose of regenerative medicine must meet the highest possible standards for both quantity and quality of transplanted cells. Cell therapies are extremely promising in respect to many diseases, both inherited and acquired. Many cell types proposed as the source for potential

Correspondence address: Prof. M. Kurpisz

Institute of Human Genetics Polish Academy of Sciences

Strzeszynska 32, 60-479 Poznan, Poland

e-mail: kurpimac@man.poznan.pl cell replacement therapy, among them the mesenchymal stem cells, embryonal stem cells (ESC) induced pluripotent stem cells (iPSC) and skeletal muscle stem cells, i.e. myoblasts, have been listed [1-4].

Satellite cells are located between the basal membrane of skeletal muscle fibres and sarcolemma. In the case of injury or intense physical activity satellite cells are activated and subsequently proliferate to form new and/or fuse with already existing myocytes forming the new muscle fibres [5]. After isolation from muscle biopsy and during in vitro propagation, satellite cells became activated and then they are named myoblasts. Myogenic origin of such myoblasts allows them to be potentially used to regenerate exhausted 
skeletal muscle fibres in muscular dystrophies or to support infarcted myocardium as well as improve dysfunctional anal and/or bladder sphincters [6-9]. For over a decade preclinical and clinical trials were conducted concerning the use of different types of stem cells; however, the obtained results did not meet the expectations, probably due to delivery of insufficient number of transplanted cells. Limited cell numbers obtained from a single donor patient and complexity of anchorage-dependent in vitro cell culture systems are among the unresolved issues. Number of myoblasts required for cell therapies are organ dependent and varies from $10^{9}$ cells (treatment of ischaemic heart) to $10^{11}$ cells in muscular dystrophies $[10,11]$. Such high number of myoblasts in T-flask in vitro culture (with density about $10^{5}$ cells $/ \mathrm{cm}^{2}$ ) requires an area approx. $10-100 \mathrm{~m}^{2}$. The organization of such a big in vitro myoblast culture system would present an enormous logistical task and extremely increase the costs of cell therapy [12].

Early data indicated that the use of Cytodex $3{ }^{\circledR}$ microcarriers in conjunction with the cell spinner bioreactors could be the optimal solution for obtaining both significant cell numbers with less financial resources and better organization of in vitro culture laboratory. Collagen-coated dextran carriers $\left(\right.$ Cytodex $\left.3^{\circledR}\right)$ were tested with several cell types, i.e. hepatocytes, chondrocytes or mesenchymal stem cells and myoblasts [13]. Area offered by microcarriers is $2700 \mathrm{~cm}^{2}$ for $1 \mathrm{~g}$ dry weight, thus relatively small amounts of beads and medium could be sufficient to obtain adequate surface to scale-up the adherent cells in in vitro culture.

The transplanted cell population should have the best possible characteristics because the in situ (recipient organ) conditions are usually sub-optimal and the ability of cells to anchor in the appropriate cell niche may greatly influence the therapy efficacy.

The aim of this study was to evaluate the proliferative potential and percentage of cells with aging markers in human primary myoblasts in vitro cultures using both cell spinner bioreactor with Cytodex $3^{\circledR}$ microcarriers and traditional T-flask culture system.

\section{Material and methods}

In vitro culture of myoblasts. Human satellite cells were isolated from skeletal muscle of adult persons obtained during the anterior cruciate ligament reconstruction as a post-operative medical waste and prepared as previously described [14]. For expansion, cells were cultured in parallel using standard T-flask $\left(75 \mathrm{~cm}^{2}\right)$ or with dextran microcarriers (GE-Amersham, Little Chalfont, UK) in the cell spinner flask - Cellspin (Integra, Plainsboro, NJ, USA). Carriers were prepared and sterilized according to the manufacturer's recommendation, then they were introduced to the culture medium at a concentration of $3 \mathrm{~g} / \mathrm{L}$. T-flasks were used for cell propagation and maintenance until the cell numbers reached amount appropriate for designed experiments. Myoblasts were cultivated in DMEM medium (Lonza, Basel, Switzerland) with $20 \%$ FBS (foetal bovine serum), $2 \mathrm{mM}$ alanyl-L-glutamine, 1\% Pen/Strep/Amphotericin B (Lonza), 1\% Chick Embryo Extract (CEE, SeraLab, West Sussex, UK), and supplemented with fibroblast growth factor bFGF (Sigma-Aldrich, St. Louis, MO, USA). The in vitro culture was carried out in medium at $37^{\circ} \mathrm{C}$ and $5 \% \mathrm{CO}_{2}$ concentration, and the cells were kept in standard T-flasks. After obtaining the appropriate number of cells, myoblasts were inoculated to microcarriers resuspended in culture media in bioreactor $\left(10^{7}\right.$ of cells per $100 \mathrm{~mL}$, i.e. $12.5 \times 10^{4} / \mathrm{cm}^{2} \mathrm{Cy}-$ todex $3^{\circledR}$ carrier surface). Then, agitation cycles started. The "adhesion" program included agitation cycle $20 \mathrm{rpm} / 5 \mathrm{~min}$ followed by $30 \mathrm{~min}$ rest cycle without agitation, subsequent "proliferation" program was based on continuous agitation with $30 \mathrm{rpm} / \mathrm{min}$ speed. Conventional (standard) in vitro cell culture with adherent cells was carried out simultaneously in order to make the appropriate comparisons.

To confirm myogenic potential of obtained cell populations, cell samples were evaluated for desmin expression by immunocytochemistry and for CD56 surface marker by flow cytometry according to previously described protocols [15]. In order to identify the cell populations following abbreviations were used: (i) 2D-Myo-D0 (starting cell population), (ii) 2D-Myo-D14 (cell population obtained after 14 days of standard T-flask culture), and (iii) 3D-Myo-D14 (cell population obtained after 14 days of microcarrier culture).

Cell cycle analysis. Cells were centrifuged at $300 \mathrm{~g}, 10 \mathrm{~min}$ at room temperature. The pellets containing $10^{6}$ cells were resuspended in $1 \mathrm{~mL}$ NIM-DAPI solution (Beckmann-Coulter, Brea, CA, USA). Samples were incubated for $10 \mathrm{~min}$ on ice in the dark and then analysed in a flow cytometer (Cell Lab Quanta cytometer, Beckmann-Coulter).

Real-time PCR. RNA isolation was performed using TRI Reagent ${ }^{\circledR}$ Reagent (Sigma-Aldrich) according to manufacturer's protocol. mRNA isolation was performed using magnetic Dynabeads ${ }^{\circledast}$ Oligo (dT) 25 (Thermofisher, Waltham, MA USA) and resuspended in $10.5 \mu \mathrm{L}$ of DEPC-water. Then reverse transcription reaction was carried out. qRT-PCR for MYOG and ACTB (reference gene) was performed on 96-well plates. Single reaction volume sample was $25 \mu \mathrm{L}$ and contained: $2 \mu \mathrm{L}$ of cDNA, $12.5 \mu \mathrm{L}$ of $2 \times \mathrm{iQ}^{\mathrm{TM}} \mathrm{SYBR}^{\circledast}$ Green Supermix (BIO-RAD, USA) and $2.5 \mu \mathrm{L} 4 \mu \mathrm{M}$ of each primer. The reaction was performed using iCycler ${ }^{\circledast}$ IQ5 (BIO-RAD, Hercules, CA, USA) and SYBR Green dye. Primers used were as following: ACTB-F 5'-CTTCCTGGGCATGGAGTCC-3'; 
ACTB-R 5'-ATCTTGATCTTCATTGTGCTG-3'; MYOG-F 5'-GCTGTATGAGACATCCCCCTA-3'; MYOG-R 5' CGACTTCCTCTTACACACCTTAC-3'

Telomere length analysis. QIAamp DNA Mini Kit was used to isolate genomic DNA (Qiagen, Hilden, Germany). The analysis was performed using a telomere TeloTAGGG Length Assay (Roche, Basel, Switzerland). Enzymatic digestion of genomic DNA with the HinfI and RsaI enzymes was carried out with subsequent electrophoretic separation of DNA fragments in $0.8 \%$ agarose gel. After denaturation and neutralization procedures, DNA overnight transfer with $20 \times$ SSC buffer to a nylon membrane was conducted. DNA was cross-linked through a two-minute exposure to UV light $(\lambda=312 \mathrm{~nm})$. Hybridization solution contained the specific DIG-labelled telomeric probe. Hybridization was carried out for $3 \mathrm{~h}$ at $42^{\circ} \mathrm{C}$. Anti-DIG-AP was added for $30 \mathrm{~min}$ incubation. AP substrate CDP-star was applied to ensure chemiluminescence reaction and the membrane was exposed to X-ray film (GE-Amersham). The signal analysis used free ImageJ software (Wayne Rasband, National Institutes of Health, Bethesda, MD USA) to determine the mean TRF value (Terminal Restriction Fragment). This value was calculated with the formula:

$$
\overline{T R F}=\frac{\sum\left(\mathrm{OD}_{\mathrm{i}}\right)}{\sum\left(\frac{\mathrm{OD}_{\mathrm{i}}}{\mathrm{L}_{\mathrm{i}}}\right)}
$$

where $O D_{i}$ value corresponds to the chemiluminescence signal and $L_{i}$ is length of TRF at $i$ position.

Cytochemical staining with SA-beta-galactosidase to assess cell aging. The procedure was performed using Cell Aging Senescence Detection Kit (BioVision, Milpitas, CA, USA). Passaged $3 \times 10^{5}$ myoblasts harvested at the time of analysis were seeded in a culture 6-well dish in the standard culture medium. Next, cells were cultured for 2 days, fixed in fixative solution (provided by manufacturer - BioVision, USA) and subjected to overnight incubation at $37^{\circ} \mathrm{C}$ in an $\mathrm{X}-\mathrm{Gal}$ staining solution (Staining Solution Mix). Additionally, cells were counterstained with eosin and evaluated under the Olympus BX40 light microscope (Olympus, Tokyo, Japan). Three populations of cells were evaluated: I — negative for SA- $\beta$-Gal; II - moderately stained cells; III — cells with strong expression of SA- $\beta$-Gal. Free ImageJ software was used to analyse obtained data.

Immunofluorescence staining for Ki67 and H2A.X markers. Cells were fixed in $4 \%$ buffered formaldehyde solution. Cell membranes were permeabilized using $0.2 \%$ solution of Triton X-100. Non-specific epitopes blocking was performed during incubation for $30 \mathrm{~min}$ in $10 \%$ goat serum, GS (Sigma-Aldrich). For the analysis polyclonal rabbit anti-Ki67 (Abcam) and mouse anti-H2A.X (Abcam) diluted at
1:500 in 10\% GS were used. Both types of antibodies were incubated overnight in $4^{\circ} \mathrm{C}$. For Ki67, a sheep anti-rabbit polyclonal TxRed-conjugated antibody was used (1:200; Abcam) and for H2A.X we used a goat anti-mouse polyclonal FITC-conjugated antibody (1:4000; Abcam).The samples were counterstained with DAPI. Slides were observed and analysed under a fluorescence microscope Imager.D1 (Carl Zeiss, Jena, Germany). For H2A.X the number of foci per nucleus were counted and based on recognition of three cell population: negative for H2A.X; with less than 5 foci per nucleus $(\mathrm{H} 2 \mathrm{~A} . \mathrm{X}<5)$; with 5 or more than 5 foci per nucleus $(\mathrm{H} 2 \mathrm{~A} . \mathrm{X}>5)$.

Apoptosis evaluation (Annexin V and PI staining). The analysis was performed using Annexin V-FITC Kit (BeckmannCoulter) according to manufacturer's recommendation. The cells were resuspended in the reaction mixture containing FITC-conjugated Annexin V and Propidium Iodide. Cell samples were incubated for $15 \mathrm{~min}$ on ice in the dark. After incubation, samples were evaluated on the Cell Lab Quanta cytometer (Beckmann-Coulter).

Statistical analysis. For all experiments statistical analyses were feasible and conducted in three replicates. The results were evaluated for normal distribution using Shapiro-Wilk test. In all cases data were calculated using ANNOVA together with Tukey multiple comparison post-tests and with p-value being adjusted for multiple comparisons. All the results (if not otherwise stated) are presented as mean \pm SEM.

\section{Results}

Comprehensive set of tests was developed to verify biological characteristics of primary human myoblast populations in two distinct in vitro cell culture systems tested. The obtained cell populations were assayed for telomere length, marker of cell aging (SA- $\beta$-galactosidase) and the cell proliferation marker (Ki67). Also the presence of apoptotic cells was evaluated and the cell cycle analysis was performed.

A diagram presenting in vitro cell propagation using both Cytodex $3^{\circledast}$ (3D-Myo-D14) and T-flask (2D-Myo-D14) cultures is shown in Figure 1. Starting myoblast population (2D-Myo-D0) myogenic marker expression is depicted in Figure 2 presenting $71.7 \%$ of desmin positive cells and $63.3 \%$ cells positive for CD56.

Cells were allowed to adhere on Cytodex $3^{\circledR}$ beads for about 3 hours. After this time, most of the cells were found to settle on dextran microcarriers and thus showed characteristic elongated shape (Figure 3A). We used a starting cell population of $2 \times 10^{6}$ myoblasts (2D-Myo-D0) and in 2 week culture we observed $4.5 \times 10^{7}$ cells in standard T-flasks (2D-Myo-D14). On 


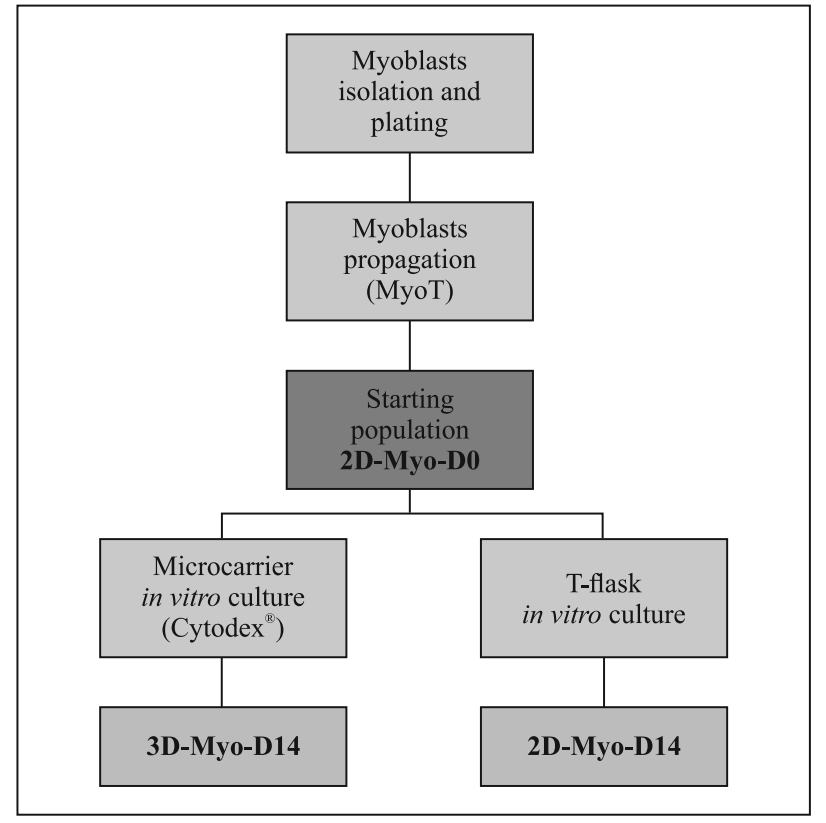

Figure 1. Diagram presenting tested systems of in vitro cultures for (i) 2D-Myo-D0 (starting cell population), (ii) 2D-Myo-D14 (cell population obtained after 14 days of standard T-flask culture), and (iii) 3D-Myo-D14 (cell population obtained after 14 days of microcarrier culture). the contrary, cells cultivated on microcarriers (with starting population $10^{7}$ ) only doubled initial population size (3D-Myo-D14 - $2.2 \times 10^{7}$ cells) (Figure 3B).

The cell cycle data showed that among the myoblasts cultured in spinner flask (3D-Myo-D14) there was a greater number of G0/G1 phase cells $(\mathrm{p}<0.0001$; Figure 4A) in contrast to the cells cultured in T-flask (2D-Myo-D14), which showed characteristics similar to the initial cell population.

Telomere length analysis showed that culture systems (2D-Myo-D14 vs. 3D-Myo-D14) did not reveal any significant effect concerning telomere length, although as expected the telomere sequences underwent shortening during subsequent days of in vitro culture (Figure 4B).

The evaluation of cell proliferation marker, Ki67 expression, showed that the number of proliferating cells was significantly decreased in both culture systems tested (17\% positive cells in the starting population (2D-Myo-D0), $3.44 \%$ in spinner cells (3D-Myo-D14) and $4.67 \%$ in T-flask culture (2D-Myo-D14) as shown in Figure 4C. Moreover, expression of myogenin marker of myogenic cells cycle exit (MYOG) was augmented

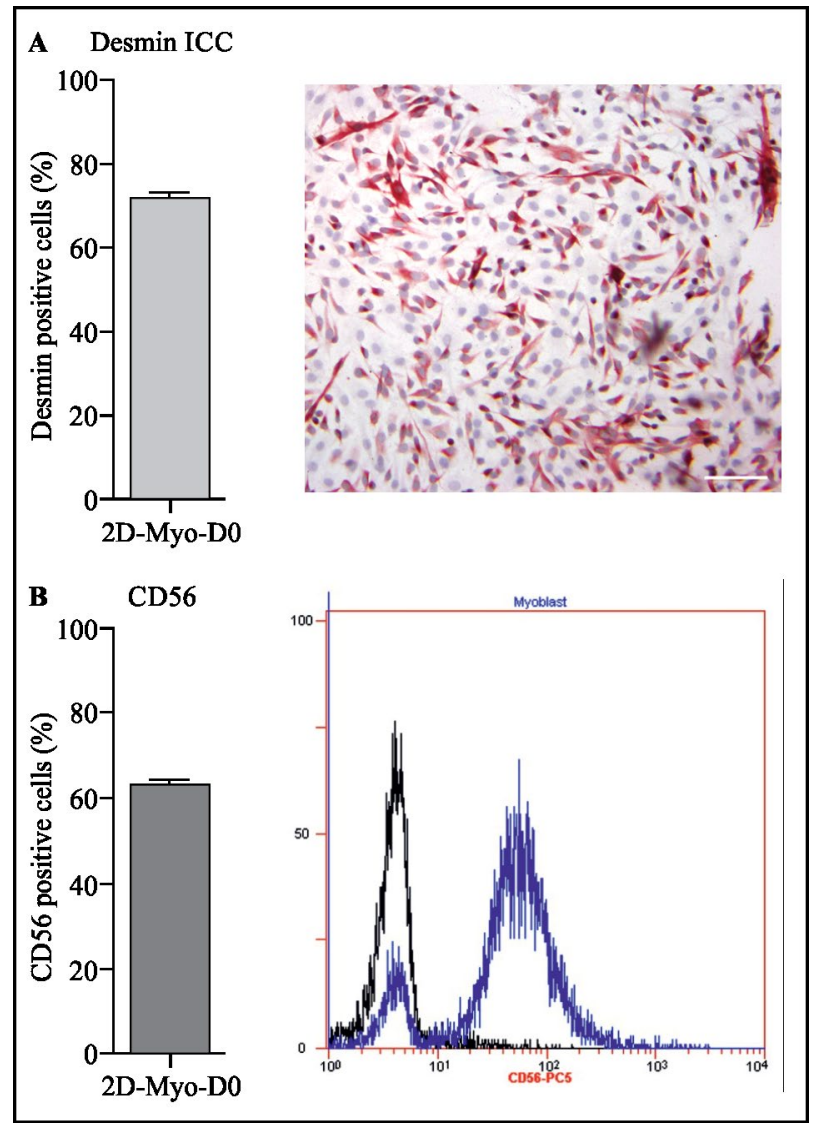

Figure 2. The starting population of myoblast (2D-Myo-D0) in respect to standard myogenic markers. A. Immunocytochemical staining for desmin (red). Nuclei counterstained with hematoxilin (blue); scale bar $-20 \mu \mathrm{m}$; B. Flow cytometry analysis of CD56 antigen expression on human myoblasts (2D-Myo-D0). Isotype control in black; anti-CD56 in blue. 


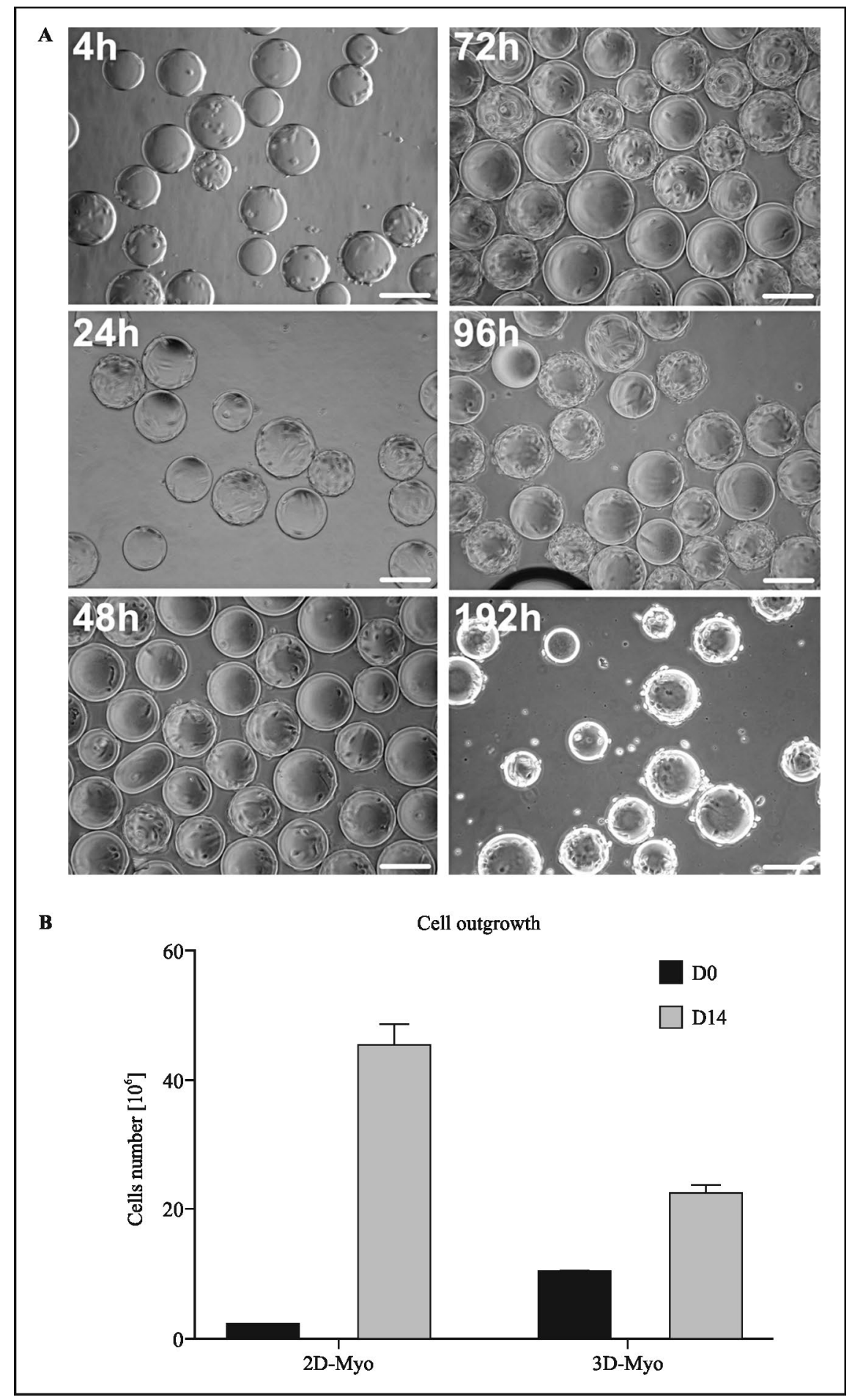

Figure 3. Microcarrier based in vitro culture system is less efficient in supporting cell proliferation compared to T-flask system. A. Myoblast samples after 4 h, 24 h, 48 h, 72 h, 96 h and 192 h of incubation on dextran microcarriers (Cytodex $\left.3^{\circledR}\right)$. Scale bars - $150 \mu \mathrm{m}$; B. Cells numbers during in vitro propagation in T-flask (2D-Myo-D14) and spinner (3D-Myo-D14) cultures.

during longer period of in vitro culture (Figure 4D: 2D-Myo-D0 vs. 2D-Myo-D14, $\mathrm{p}<0.01$; 2D-Myo-D0 $v s$. 3D-Myo-D14, $\mathrm{p}<0.001$ ). We also observed that cells cultured on microcarriers showed higher ex- pression of MYOG (2D-Myo-D14 vs. 3D-Myo-D14, $\mathrm{p}<0.05)$.

As illustrated in Figure 5A in both culture systems, the cells were found positive for the $\beta$-galactosidase-SA. 


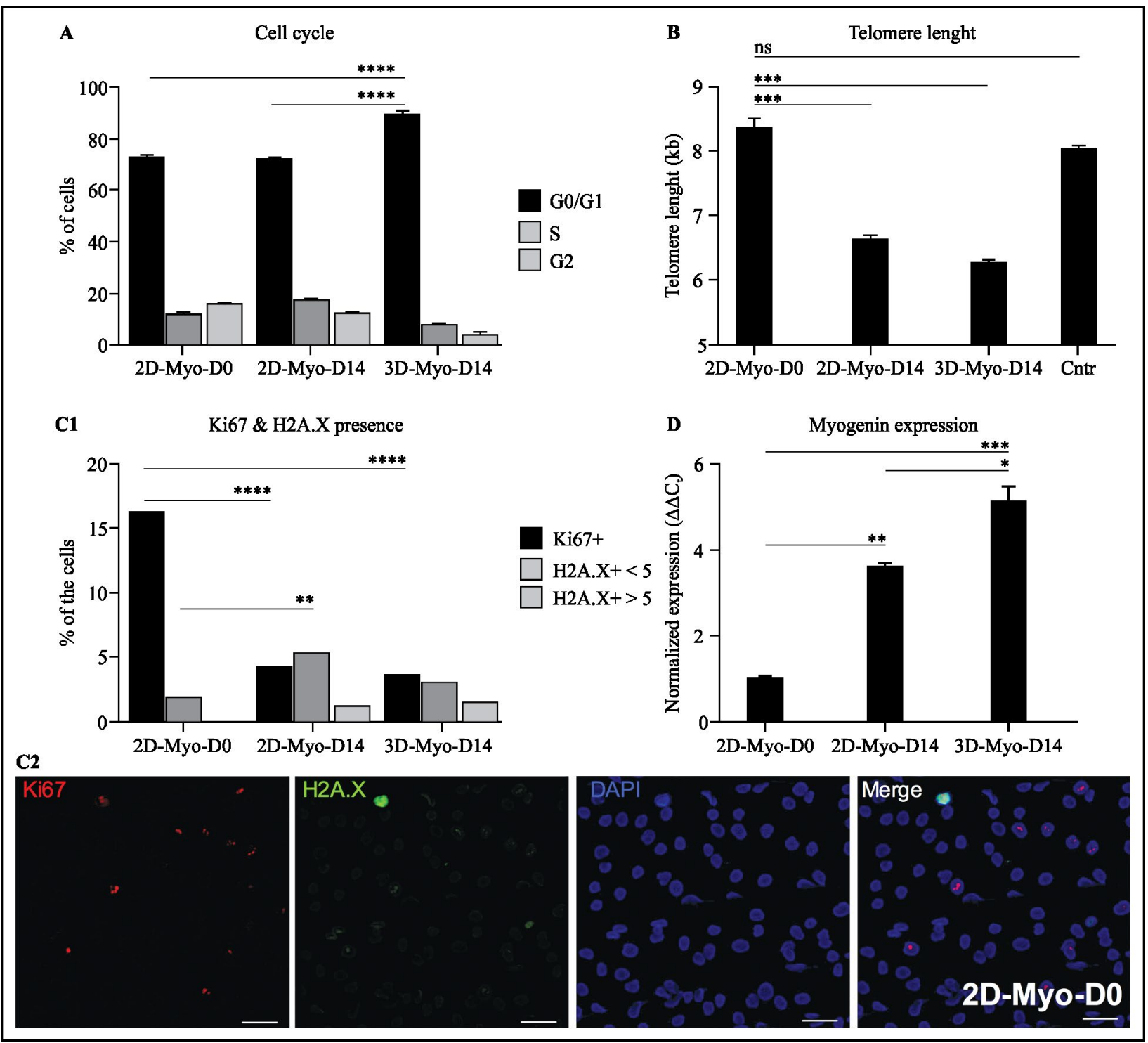

Figure 4. Comparison of proliferation potential of myoblasts cultured in vitro on dextran microcarriers (3D-Myo-D14) and monolayer based T-flask (2D-Myo-D14) to starting cell population (2D-Myo-D0). A. Flow cytometry evaluation of the cell cycle distribution within analyzed myoblast populations (3D-Myo-D14 vs. 2D-Myo-D0, p < 0.0001); B. Southern blot analysis of myoblasts telomere length on d0 and after 14 days in both system of $i n$ vitro culture (2D-Myo-D0, p < 0.001); C1. Ki67 (proliferation marker) and H2A.X (DNA damage marker/double strand break) immunofluorescence evaluation; C2. Immunofluorescence staining of Ki67 (red) and H2A.X (green) in myoblast starting population (2D-Myo-D0), counterstained with DAPI (blue). Scale bar $-10 \mu \mathrm{m}$; D. Myogenin expression after long term in vitro myoblast culture in both culture systems in comparison to starting myoblast population $(\mathrm{p}<0.001)$.

However, in myoblasts cultured in the cell spinner (3D-Myo-D14) differences were more significantly expressed; negatively-stained cell population was diminished while strongly-positive (aged) cells appeared (3D-Myo-D14 vs. 2D-Myo-D14 at $\mathrm{p}<0.0001$ ).

The presence of apoptotic cells was assessed using FITC-Annexin and Propidium Iodide (PI) staining followed by flow cytometry evaluation
(Figure 5B). Reduced viable cell numbers in the populations tested $v s$. starting cell population (2D-Myo-D0) were observed (2D-Myo-D0 - 99.4\%, 2D-Myo-D14 - 88.4\% and 3D-Myo-D14 - 69.7\%) showing significant differences $(p<0.0001)$. Significant numbers of dead cells were visible in 3D-Myo-D14 system in comparison to standard T-flask culture $(\mathrm{p}<0.01)$. 


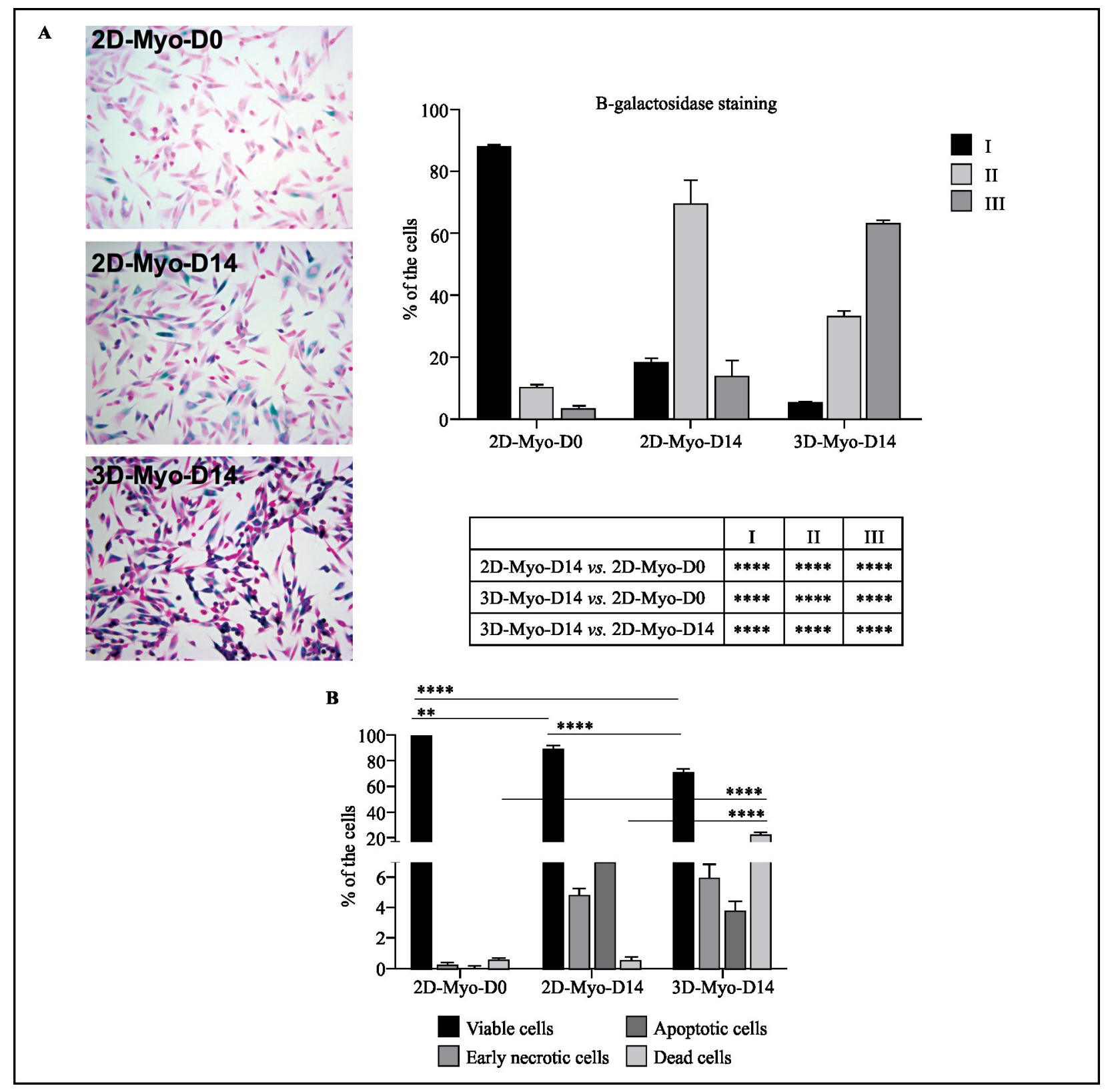

Figure 5. Apoptosis and cell senescence evaluation in myoblast populations under study. A. SA- $\beta$-galactosidase (SA- $\beta$-Gal) expression in tested in vitro culture system. Three distinct populations were clearly seen: negative for SA- $\beta$-Gal cells (I), moderately stained (II) and strongly stained cells (III); scale bar $-20 \mu \mathrm{m}$; B. Viable and apoptotic cells numbers in both culture systems were compared (2D-Myo-D0 vs. 2D-Myo-D14, p < 0.001, and 2D-Myo-D0 vs. 3D-Myo-D14, p < 0.0001). Dead cells population in 3D-Myo-D14 reached over $20 \%$ and was significantly higher than in the other analyzed myoblast populations.

\section{Discussion}

The aim of this study was to evaluate a scaling up process efficacy of primary myoblast cultures comparing traditional (T-flasks) means of monolayer in vitro culture and in parallel studied collagen-coated-dextran microcarriers with concomitant spinning (bioreactor). This 3D-suspension in vitro culture attempt could potentially enable to procure large cell numbers lowering culture costs in opposition to standard T-flask-based laborious procedure. So far, myoblast microcarrier cultures were performed solely using established mouse cell lines (C2C12) or by using human myoblasts obtained from the young individual $[10,16]$. High proliferative potential of mouse $\mathrm{C} 2 \mathrm{C} 12$ cell line is widely recognized and un- 
fortunately it is not comparable with human primary myoblast suspension characteristics, therefore in many cases it should not be used as a model relevant for primary human myoblasts. Moreover, it is known that human myoblast proliferative potential significantly decreases with age [17]. The individual's age seems to be the key factor for patient to whom stem cell therapy is proposed. In our experiments the cells obtained from adult donors in the Cytodex $3^{\circledast}$ based in vitro culture failed to sustain an active proliferation in relation to the standard T-flask conditions. The reasons could be found in both the greater sensitivity of adult cells to shear forces operations in the stirring process or/and reduced the strength of myoblasts adhesion to the dextran microcarriers comparing to T-flask surface [14]. In previous experiments there was described the phenomenon of log-phase entering during myoblast microcarriers cultures [10]. It could cause the inhibition of cell proliferation for the first 10 days of culture and only after this time point one could have noticed an increase in the cell number. However, in those experiments myoblasts derived originated from a very young donor and according to our group long-term observations (over 15 years) the patient age has a strong influence on the quantity and quality of myoblasts obtained (data not shown). It could be speculated that the infant-derived cells were able to adapt to microcarriers culture conditions on the contrary to the cells isolated from adult muscle tissue. Moreover, previous studies did not assess cell characteristics in detail (i.e. apoptotic or senescence cell markers etc.).

In respect to myoblasts telomeres shortening data their length was not related to the culture system here applied. It has been previously demonstrated that myogenic cell line of immortalized mouse myoblasts (C2C12) showed some telomerase activity [18], while mouse satellite cells were telomerase positive only in in vivo conditions. However, human myoblasts lost their telomerase activity in in vitro culture within a few days [16]. It is likely to be a result of accelerated cell differentiation rather than the cell senescence [19]. Optimizing the conditions of human myoblast isolation and in vitro culture for regenerative medicine purpose it could be beneficial not only to obtain cell populations with high telomerase activity, but one can consider to deliver signals for augmentation of telomerase expression until the requested numbers of cells are achieved. However, the oncogenic potential of cells with high telomerase activity should be also considered. A change of the culture conditions to diminish telomerase activity before cell transplantation could be therefore recommended. Replicative cell aging is described in the literature as state, where cells enter G0 phase, cease proliferation and exhibit number of changes in gene expression profile, morphology or cytophysiology. In agreement with previously obtained data, we have also observed a decreased number of myoblasts in in vitro culture presenting Ki67 proliferation marker. This may indicate acceleration in reaching the Hayflick limit of cell population doubling while maintaining them on microcarriers with declining proliferative potential together and advanced replicative aging [20]. Evaluation of myogenin expression determined by real-time PCR confirmed the results previously obtained in which the Northern Blot technique was used. Present study showed a significant increase in MYOG expression during in vitro culture of cells on microcarriers (3D-Myo-D14). This confirms the observation that long-term culture of human myoblasts does not lead to the loss of their myogenic potential but it seems that the cells go through successive stages of differentiation during extended in vitro propagation. Moreover, the myogenin expression has been also discussed as a first marker towards establishment of post mitotic state of differentiating myoblasts [21]. Some authors indicate that mechanical stimulation via laminin receptors leads to increase of myogenin expression and induces myotube formation. In our experiments, Cytodex ${ }^{\circledR} 3$ anchored cells experienced much stronger mechanical stress due to beads binding and medium stirring so upregulation of myogenin expression could have occurred [22]. The presence of $\beta$-galactosidase enzyme (SA-beta-Gal) could be correlated with the cells senescence status in in vitro culture [23]. Also in this case, the conditions provided by standard T-flask culture were more favourable than those in the cell spinner system.

The hostile culture environment of spinner caused further reduction of cell viability with significantly increasing population of dead cells. Application of stirring cycles can hamper cell adhesion and shear forces could have also led to the observed cell loss. Incidentally, culture conditions favouring the replicative aging do not seem to be beneficial and justified for stem cell propagation. Myoblast cell therapy can be also potentially offered to dystrophic patients. It is well documented that muscular dystrophies are frequently linked to premature cell aging and exhaustion of satellite cells pool [24] so dystrophic myoblasts obtained from relatively young individuals should be considered as the adult ones demonstrating of diminished proliferative potential.

The present study shows that despite the encouraging results of in vitro bioreactor approach pursued by the other research groups employing either the mouse myoblast immortalized $\mathrm{C} 2 \mathrm{C} 12$ line or infant-derived 
human primary myoblast population, adult human skeletal myoblasts microcarrier culture exhibited a number of serious disadvantages. Therefore, we tend to think that this is the first report describing the impact of spinner 3D-type of culture conditions on adult human myoblast viability.

Faster replicative aging and reduced number of proliferating cells raise the doubts in applicability of this cell culture system for myogenic origin stem cells. Because myoblasts are extraordinary promising as a source of cells in their potential application in regenerative medicine there should be further pursued continuing efforts towards the optimization of culture medium and/or its supplements. Since the main goal is to create the conditions similar to the satellite cells niche in vivo in order to support telomerase activity thus obtaining the cell population with increased proliferative potential would be desired.

Since the negative effect of the cell spinner in vitro culture has been shown here on adult primary human myoblast suspensions one could speculate the reason for such phenomenon. It should be indicated both shear forces and possibly mechanical stress could be one of the considerable reasons. There is still a strong need to create a new platform devoid of these setbacks; however, the putative new systems should also offer a more favourable free surface to culture medium volume ratio in respect to dextran microcarriers. It appears to be feasible that treatment with adhesively-dependent stem cells should be introduced as the standard procedure directed to a large number of patients.

\section{Conflict of interests}

The authors indicate no potential conflict of interests.

\section{Acknowledgements}

This work was supported by the National Science Centre Poland, grant no. 2014/13/B/NZ3/04646 and grant no. NN 401097 937; TJK is supported by the Foundation for Polish Science (FNP).

\section{References}

1. Young PP, Schäfer R. Cell-based therapies for cardiac disease: a cellular therapist's perspective. Transfusion. 2015;55:441-451. doi: 10.1111/trf.12826.

2. Fox IJ, Daley GQ, Goldman SA, Huard J, Kamp TJ, Trucco M. Stem cell therapy. Use of differentiated pluripotent stem cells as replacement therapy for treating disease. Science. 2014;345:1247391. doi: 10.1126/science.1247391.

3. Seidel M, Borczyńska A, Rozwadowska N, Kurpisz M. Cell-based therapy for heart failure: skeletal myoblasts. Cell Transplantation. 2009;18:695-707. doi: 10.3727/096368909X 470810.
4. Buzhor E, Leshansky L, Blumenthal J et al. Cell-based therapy approaches: the hope for incurable diseases. Regen Med. 2014;9:649-672. doi: 10.2217/rme.14.35.

5. Yablonka-Reuveni Z. The skeletal muscle satellite cell: still young and fascinating at 50.J Histochem Cytochem. 2011;59:1041-1059. doi: 10.1369/0022155411426780.

6. Romaniszyn M, Rozwadowska N, Nowak M et al. Successful implantation of autologous muscle-derived stem cells in treatment of faecal incontinence due to external sphincter rupture. Int J Colorectal Dis. 2013;28:1035-1036. doi: 10.1007/ s00384-013-1692-y.

7. Siminiak T, Fiszer D, Jerzykowska $\mathrm{O}$ et al. Percutaneous trans-coronary-venous transplantation of autologous skeletal myoblasts in the treatment of post-infarction myocardial contractility impairment: the POZNAN trial. Eur Heart J. 2005;26:1188-1195. doi: 10.1093/eurheartj/ehi159.

8. Peters KM, Dmochowski RR, Carr LK et al. Autologous muscle derived cells for treatment of stress urinary incontinence in women. J Urol. 2014;192:469-476. doi: 10.1016/j. juro.2014.02.047.

9. Negroni E, Bigot A, Butler-Browne GS, Trollet C, Mouly V. Cellular Therapies for Muscular Dystrophies: Frustrations and Clinical Successes. Hum Gene Ther. 2016;27:117-126. doi: 10.1089/hum.2015.139.

10. Palmieri B, Tremblay JP. Myoblast transplantation: a possible surgical treatment for a severe pediatric disease. Surg Today. 2010;40:902-908. doi: 10.1007/s00595-009-4242-z.

11. Kim J, Shen W, Gallagher D, Jones A Jr, Wang Z, Wang J, Heshka S, Heymsfield SB. Total-body skeletal muscle mass: estimation by dual-energy X-ray absorptiometry in children and adolescents. Am J Clin Nutr. 2006;84:1014-1020. PMID: 17093152.

12. Boudreault P, Tremblay JP, Pépin MF, Garnier A. Scale-up of a myoblast culture process. J Biotechnol. 2001;91:63-74. PMID: 11522363.

13. Sun LY, Lin SZ, Li YS, Harn HJ, Chiou TW. Functional cells cultured on microcarriers for use in regenerative medicine research. Cell Transplant. 2011;20:49-62. doi: 10.3727/096368910X532792.

14. Kolanowski TJ, Rozwadowska N, Malcher A et al. In vitro and in vivo characteristics of connexin 43-modified human skeletal myoblasts as candidates for prospective stem cell therapy for the failing heart. Int J Cardiol. 2014;173:55-64. doi: 10.1016/j. ijcard.2014.02.009.

15. Rozwadowska N, Kolanowski TJ, Wiland E et al. Characterization of nuclear architectural alterations during in vitro differentiation of human stem cells of myogenic origin. PLoS One. 2013;8:e73231. doi: 10.1371/journal.pone.0073231.

16. Bardouille C, Lehmann J, Heimann P, Jockusch H. Growth and differentiation of permanent and secondary mouse myogenic cell lines on microcarriers. Appl Microbiol Biotechnol. 2001;55:556-562. PMID: 11414320.

17. Wernig A, Schäfer R, Knauf U et al. On the regenerative capacity of human skeletal muscle. Artif Organs. 2005;29:192-198. PMID: 15725215.

18. Holt SE, Wright WE, Shay JW. Regulation of telomerase activity in immortal cell lines. Mol Cell Biol. 1996;16:2932-2939. PMID: 8649404.

19. O'Connor MS, Carlson ME, Conboy IM. Differentiation rather than aging of muscle stem cells abolishes their telomerase activity. Biotechnol Prog. 2009;25:1130-1137. doi: 10.1002/btpr.223.

20. Shiomi K, Kiyono T, Okamura K et al. CDK4 and cyclin D1 allow human myogenic cells to recapture growth property 
without compromising differentiation potential. Gene Ther. 2011;18:857-866. doi: 10.1038/gt.2011.44.

21. Andrés V, Walsh K. Myogenin expression, cell cycle withdrawal, and phenotypic differentiation are temporally separable events that precede cell fusion upon myogenesis. J Cell Biol. 1996;132:657-666. PMID: 8647896.

22. Grossi A, Yadav K, Lawson MA. Mechanical stimulation increases proliferation, differentiation and protein expression in culture: stimulation effects are substrate dependent. J Biomech. 2007;40:3354-3362. PMID: 17582421.
23. Ksiazek K, Mikuła-Pietrasik J, Jörres A, Witowski J. Oxidative stress-mediated early senescence contributes to the short replicative life span of human peritoneal mesothelial cells. Free Radic Biol Med. 2008;45:460-467. doi: 10.1016/j. freeradbiomed.2008.04.032.

24. Malatesta M, Giagnacovo M, Renna LV, Cardani R, Meola G, Pellicciari C. Cultured myoblasts from patients affected by myotonic dystrophy type 2 exhibit senescence related features: ultrastructural evidence. Eur J Histochem. 2011;55:e26. doi: 10.4081/ejh.2011.e26.

Submitted: 19 November, 2015 Accepted after reviews: 23 May, 2016

Available as AoP: 7 June, 2016 